\title{
Free-volume correlations in positron-sensitive annihilation modes in chalcogenide vitreous semiconductors: on the path from illusions towards realistic physical description
}

\author{
O.I. Shpotyuk ${ }^{1, *}$, M.M. Vakiv ${ }^{1}$, M.V. Shpotyuk ${ }^{1,2}$, A. Ingram $^{3}$, J. Filipecki ${ }^{4}$, A.P. Vaskiv ${ }^{5}$ \\ ${ }^{\prime}$ Institute of Materials of SRC „,Carat”, 202, Stryjska str., 79031 Lviv, Ukraine \\ ${ }^{2}$ Lviv Polytechnic National University, 12, Bandera str., 79013 Lviv, Ukraine \\ ${ }^{3}$ Opole University of Technology, 75, Ozimska str., Opole, 45370, Poland \\ ${ }^{4}$ Institute of Physics of Jan Dlugosz University, \\ 13/15, al. Armii Krajowej, Czestochowa, 42200, Poland \\ ${ }^{5}$ Ivan Franko Lviv National University, 6, Kyryla \& Mefodia str., 79005 Lviv, Ukraine \\ *The corresponding author e-mail: shpotyuk@novas.lviv.ua
}

\begin{abstract}
A newly modified correlation equation between defect-related positron lifetime $\tau_{2}$ (ns) defined within two-state model and corresponding radius $R(\AA)$ of freevolume positron traps in the full non-linear form$$
\boldsymbol{\tau}_{\mathbf{2}}=0.285 \cdot\left[1-\frac{R}{R+5.50}+\frac{1}{2 \pi} \cdot \sin \left(\frac{2 \pi R}{R+5.50}\right)\right]^{-1}
$$$$
\text { or simplified linear-approximated form }
$$$$
\boldsymbol{\tau}_{\mathbf{2}}=0.212 \cdot(1+0.244 \cdot R)
$$

is proved to account for compositional trends in void volume evolution of chalcogenide semiconductor compounds like binary As-S(Se) glasses. Specific chemical environment of free-volume voids associated with neighbouring network-forming polyhedrons is shown to play a decisive role in this correlation, leading to systematically enhanced estimated void sizes in comparison with typical molecular substrates, such as polymers.
\end{abstract}

Keywords: chalcogenide glass, free volume, positron annihilation, positronium, lifetime, trapping defect.

Manuscript received 05.05.14; revised version received 22.08.14; accepted for publication 16.09.14; published online 30.09.14.

\section{Introduction}

In recent decades, the positron annihilation lifetime (PAL) spectroscopy becomes an important noninvasive technique for routinely probing the atomic-deficient free-volume arrangement in a great variety of high-tech semiconductor materials irrespectively to their structural organization [1-3]. Recently, this method has been employed even for such complicated disordered solids as chalcogenide vitreous semiconductors (ChVS) [3-10]. These glassy-like alloys of chemical elements from IV-V groups of the Periodic table (typically As, $\mathrm{Ge}, \mathrm{Sb}, \mathrm{Bi}$, etc.) with chalcogen atoms ( $\mathrm{S}, \mathrm{Se}$ or $\mathrm{Te}$ ) prepared by rapid melt-quenching [11] is one of the most promising functional media for IR photonics covering both atmospheric and space telecommunication windows [12-14]. Owing to their rich atomic-deficient structure, ChVS allow an unprecedented possibility to accommodate different host additives ensuring unique non-linear optical functionality in a wide spectral region $[14,15]$. Nevertheless, the possibilities of PAL technique, especially those governed by PAL-sensitive structureproperties correlations, are often treated too ambiguously in application to different ChVS systems, without relation with realistic physical picture.

To a great extent, it has been demonstrated by changes in defect-related positron lifetime $\tau_{2}$ as 
influenced by structural features of ChVS-based glassforming networks. In general, such lifetimes for canonical two-state model with one preferential type of positron trapping structural defects can be calculated by direct solving the Schrödinger equation for given electron-positron interaction model with known crystal potential $[1,2]$. Electronic states of positrons identified within density functional theory (DFT) allows calculations of defect-related positron lifetimes $\tau_{2}$, which are very close to the realistic ones in the dependence on correctness of the chosen crystal potential. This calculation procedure can serve as a key enabling the expected PAL modes in semiconductors, where direct experimental tests have not been carried out yet or they have been impossible at all in view of some technological constraints in materials preparation [1].

Other approach utilizes a specific case of correlation relationship between long-lived lifetime $\tau_{3}$ in the decomposed PAL spectra, which is associated with decaying of bounded electron-positron states of positronium Ps atoms, and corresponding radius $R$ of free-volume void, where Ps is either quenched or inhibited by interaction with nearest chemical environment $[1,2]$. Under this condition, the Ps annihilation lifetime $\tau_{3}$ can be substituted by defectrelated positron lifetime $\tau_{2}$ extracted from the same twostate trapping model, provided both positron and o-Ps are trapped before annihilation similarly [16-21]. So, this method gives a rough estimation procedure, which allows only evaluate an expected void size in solids within a sequent $\tau_{3}-\tau_{2}-R$ correlation, the void characteristics being considerably dependent on preferential chemistry of positron trapping sites.

In spite of obvious difference between both these methods, a crude speculative attempt to ignore the first calculation approach in a favor of the second estimation one has been made recently [22, 23]. Thus, in [23], the modified sequent $\tau_{3}-\tau_{2}-R$ correlation was utilized as a basis of the generalized void-size-determination procedure for a wide range of materials, involving molecular substrates, such as polymers, as well as inorganic disordered solids like ChVS. Ignoring the principal difference in the chemical nature of these materials led to evidently misleading conclusion on expected positron trapping void volumes, which occur to be in apparent contradiction with proved experimental data for a great variety of ChVS [3-10]. The time is come to analyze critically these discussible diversities, asking a simple question: "How far can we really go with free-volume parameterization in amorphous semiconductors such as ChVS aided by PAL technique?". With this in mind, in this work, we present a critical assessment on methodological possibilities of PAL-related structure-properties correlations in different types of materials, varying substantially their structural organization but in strong respect to their specifics chemistry, so to be calibrated with ChVS exemplified by binary As-S/Se network glass formers.

\section{On the applicability of Liao's et al. [16] approach as a determinant of sequent $\tau_{3}-\tau_{2}-R$ correlation}

Under twinned condition that (1) Ps is either quenched or inhibited by interaction with nearest chemical environment, and (2) positron and Ps trapping in the medium is seemingly governed, the Ps lifetime $\tau_{3}$ can be simply substituted by defect-related positron lifetime $\tau_{2}$ extracted from the canonical two-state trapping model [1-3]. It means that the well-known relationship between the void radius $R$ and o-Ps lifetime $\tau_{3}$ in a spherical infinitive potential model by Tao S.J. [24] with positron and electron wave functions overlapped through homogeneous $\Delta R$ layer can be used to determine the $\tau_{2}$ $R$ correlation. It was firstly fitted with void sizes in molecular and zeolite systems to reflect the case of intrinsic vacuum decay [25]. Under essential effect of chemical environment in positron-electron interaction, this $\tau_{2}-R$ correlation can be simply parameterized in respect to the generalized master equation below with material-related parameters $C$ and $\Delta R$ [16-21]:

$\tau_{2}=C \cdot\left[1-\frac{R}{R+\Delta R}+\frac{1}{2 \pi} \cdot \sin \left(\frac{2 \pi R}{R+\Delta R}\right)\right]^{-1}$.

Both parameters $C$ and $\Delta R$ in Eq. (1) attain a quite deterministic physical meaning, when they are used to calculate the defect-related positron lifetimes $\tau_{2}$. In this case, $C$ converges into intrinsic defect-free bulk positron lifetime $\tau_{b}$ (e.g. experimental lifetime of positrons trapped in voids of vanishing sizes $R \rightarrow 0$ ) and $\Delta R$ approaches the electron layer thickness for positron penetration wall region of free-volume trapping void [16-21]. It is quite understandable that both parameters are entirely defined by materials chemistry of positron trapping sites. The only way to account these parameters is to parameterize master Eq. (1) semi-empirically using available experimental data on volume expansion/ compression in materials with known void sizes.

For many polymer systems of the same chemical nature, above Eq. (1) can be fitted to known experimental data giving a suitable form of this analytical correlation to calculate radii $R(\AA)$ of freevolume voids responsible for positron trapping with the lifetime $\tau_{2}$ (ns). Thus, it was shown [16] that for molecular substrates, such as polymers, the corresponding equation calibrated with known or calculated cavity sizes of some zeolites and molecular sieves can be adequately presented as:

$$
\tau_{2}=0.260 \cdot\left[1-\frac{R}{R+3.823}+\frac{1}{2 \pi} \cdot \sin \left(\frac{2 \pi R}{R+3.823}\right)\right]^{-1} .
$$

Since Eq. (2) was not the best to fit the known experimental data in the probed polymers for small positron lifetimes $\tau_{2}$ and void radii $R$ (where Ps does not appear because of geometrical constraints $[1,8,9])$, it was proposed to replace it by the simplified linear equation: 
$\tau_{2}=0.174 \cdot(1+0.494 \cdot R)$

But this latter Eq. (3) is only a rough approximation of former Eq. (2) and, as it is reasonably noticed in [16], it works only for small defect lifetimes $\tau_{2}<0.26 \mathrm{~ns}$, when Eq. (2) is not applicable.

In [22, 23], these Eqs. (2) and (3) were accepted in the same unchanged form for all other kinds of materials, in part, for ChVS, which were imagined, irrespectively to principal chalcogen environment, as full chemical analogues of typical polymers. This speculation allows incorrectly equilibrate void volumes responsible for positron trapping in ChVS and polymers [16]. Thus, the defect lifetime $\tau_{2}=0.36 \mathrm{~ns}$ in glassy g$\mathrm{As}_{2} \mathrm{~S}_{3}[3,4,7,22]$ was attributed in respect to Eq. (2) to spherical voids of only $V \approx 57 \AA^{3}$ in a volume $(R=$ $2.36 \AA)$. Further, this value was even more underestimated to $V=42 \AA^{3}(R=2.16 \AA)$ using linear Eq. (3) [22], despite $\tau_{2}$ value (0.36 ns) was clearly higher than the characteristic limit of zeolite-type environment (0.26 ns).

Not type of interatomic linking (polymeric or nonpolymeric), but only principal chemical nature of this linking plays a decisive role in the governed $\tau_{3}-\tau_{2}-R$ correlation. While ChVS form specific class of inorganic polymers possessing long inter-chain linking due to multiple chalcogen-chalcogen bonding [11], the approach [22] assuming equivalency of Eq. (2) for both organic and inorganic polymers seems simply wrong. It is quite understandable from main principles of PAL spectroscopy [1-3], that not polymer-type linking in a sense of infinite structural entities is a necessary condition for such extension, but only similarity in specific chemical environment of free-volume voids trapping positrons [16-21]. The authors [16] gives a detailed list of these chemicals exhibiting strong quenching (niroaromatics, quinones, maleic anhidrides, ions with redox potential less $0.9 \mathrm{eV}$ ) and inhibition (polyimides and materials containing only carbon) in Ps annihilation.

The sequent $\tau_{3}-\tau_{2}-R$ correlations were justified for many types of polymers as it summarized in Table 1 , the calculations for the controlled value $\tau_{2}=0.36 \mathrm{~ns}$ being performed to compare them. It is clearly seen that both fitting parameters $C$ and $\Delta R$ in master Eq. (1) attain a wide domain of variation in dependence on materials chemistry. $C$ is mainly affected by changes in the averaged value of defect-free bulk positron lifetime $\tau_{b}$, while $\Delta R$ reveals more complicated behavior in respect to changes in the overlapping layer of positron-electron wave functions. It is interesting that, even for the same type of polymers such as polystyrene, these parameters significantly differ with the applied method of void evolution (temperature- or pressure-affected changes). As a result, the estimated values of void volumes responsible for positron trapping with $\tau_{2}=0.36 \mathrm{~ns}$ (the characteristic value proper to $\left.\mathrm{g}-\mathrm{As}_{2} \mathrm{~S}_{3}[3,4,7,22]\right)$ varies dependently on polymers from 10 to $83 \AA^{3}$.
For comparison, the similar data of V.I. Grafutin et al. [26] for some inorganic substances such as crystalline $\mathrm{Si}$ and $\mathrm{Fe}$ with vacancy-type positron traps are also included to Table 1 . It seems noteworthy that, despite obvious difference in $C$ and $\Delta R$ parameters, the control void volume associated with $\tau_{2}=0.36 \mathrm{~ns}$ is relatively large and close to that as in the case of temperatureactivated positron-trapping voids in polystyrene. The greater values of free volumes responsible for positron trapping in inorganic solids as compared with preferential $\mathrm{C}-\mathrm{H}$ environment in polymers are probably related to more significant intrinsic electric fields proper to denser atomic packing.

The ChVS with their typical covalent-bonded networks built of elements from IV-VI groups of the Periodic table do not fit to any of the above groups of chemicals summarized in Table 1 . The most essential is absence of chalcogen atoms in the nearest atomic environment of potential positron traps, since they form specific cones of BFSA owing to stiff covalent bonding in both cis- and trans-configurations [27]. These voids are especially effective positron traps in the nearest surrounding of the chalcogen-pnictogen bond, when they possess preferential negative charge due to difference in the electronegativities between these atoms [28]. It means that rough extension of Eq. (1) to the final form of Eqs. (2) and/or (3), calibrated for zeolites and molecular sieves, is fully unacceptable for ChVS. So, the approach by K.-S. Liao et al. [16] cannot be simply spread for other kinds of materials (having principally different chemical environment) beyond typical molecular substrates such as polymers without essential modification of the applied master equation.

\section{On the Jensen's et al. [29] calculations for direct $\tau_{2}-R(V)$ correlation in ChVS}

Theoretical calculations of positron annihilation parameters are an important task on the path to correct identification of free-volume defects in solids using PAS methods. In general, this theory deals with solving the Schrödinger equation for adopted electron-positron interaction model to get electron and positron wave functions at the background of known crystal potential $[1,2,30]$. Electronic calculations for electron and positron states are typically performed self-consistently and simultaneously within two-dimensional DFT under corresponding choice of exchange-correlation energy (typically, in the local-density approximation (LDA)) [31-33]. Assuming full atomic superposition, i.e. no relaxation effects in a crystalline lattice, a threedimensional potential for positrons can be well constructed, which allows unrelaxed geometry for generated free-volume defects. Within this approach, the quantitative parameters of these free-volume type defects (vacancies, vacancy-like clusters and agglomerates) contributed to positron annihilation can be readily defined by removing some atoms or their groups 
Table 1. Sequent $\tau_{3}-\tau_{2}-R$ correlation parameterized in respect to master Eq. (1) for different substances with a controlled void volume $V$ (or radius $R$ in a spherical approximation) for $\tau_{2}=0.36 \mathrm{~ns}$.

\begin{tabular}{|l|c|c|l|c|c|}
\hline$\#$ & $\begin{array}{c}C, \\
\mathrm{~ns}\end{array}$ & $\begin{array}{c}\Delta R, \\
\AA\end{array}$ & \multicolumn{1}{|c|}{ Parameterized material and method } & $\begin{array}{c}V, \AA^{3}(R, \AA) \text { for } \\
\tau_{2}=0.36 \mathrm{~ns}\end{array}$ & Reference \\
\hline 1 & 0.22 & 1.66 & Amine-cured epoxy polymers, pressure effects & $10.0(1.33)$ & {$[17]$} \\
\hline 2 & 0.245 & 3.419 & Epoxy polymers, pressure effects & $54.3(2.35)$ & {$[16,18]$} \\
\hline 3 & 0.280 & 4.288 & Polystyrene, pressure effects & $52.0(2.32)$ & {$[16]$} \\
\hline 4 & 0.141 & 2.168 & Polystyrene, temperature effects & $82.6(2.70)$ & {$[16]$} \\
\hline 5 & 0.260 & 3.823 & $\begin{array}{l}\text { Molecular substrates such as polymers calibrated for spherical } \\
\text { cavities in zeolites }\end{array}$ & $56.7(2.38)$ & {$[16]$} \\
\hline 6 & 0.22 & 3.4 & Vacancies in Si crystal & $83.8(2.72)$ & {$[26]$} \\
\hline 7 & 0.11 & 1.8 & Vacancies in Fe crystal & $83.1(2.71)$ & {$[26]$} \\
\hline
\end{tabular}

from a perfect crystal structure to recalculate the corresponding positron wave functions for remaining defected geometry.

This approach was comprehensively applied to ChVS by K.O. Jensen et al. at the beginning of the 1990-s [29]. With respect to As-Se glasses that were most studied by PALS in that time due to earlier experimental works by V.P. Shantarovich with co-authors [34, 35], the bulk and defect-related positron lifetimes were calculated by approximating an orthorhombic structure for monoclinic c- $\mathrm{As}_{2} \mathrm{Se}_{3}$ at the basis of Puska's model [31-33]. These lifetimes occur to be $0.240 \mathrm{~ns}$ for nontrapped positron annihilation in defect-free bulk c$\mathrm{As}_{2} \mathrm{Se}_{3}$, while in defected crystal they reached essentially higher values $0.262,0.274,0.316$ and $0.368 \mathrm{~ns}$ for single $\mathrm{As}$ and Se vacancies, As-Se divacancy and $\mathrm{As}_{-} \mathrm{Se}_{3}$ quadruple vacancy, respectively. Thus, by accepting $\sim 25 \AA^{3}$ as a mean atomic volume in $\mathrm{As}_{2} \mathrm{Se}_{3}$, it was possible to describe dependence of positron lifetimes $\tau_{2}(\mathrm{~ns})$ on vacancy volume $V\left(\AA^{3}\right)$ in respect to the simple linear equation [3]:

$\tau \cong 0.240+0.0013 \cdot V$.

This dependence is believed to be valid in the same linear form (4) for As-S glasses, since close defect-free bulk positron lifetime $\tau_{b}=0.242 \mathrm{~ns}$ for crystalline orpiment $\mathrm{As}_{2} \mathrm{~S}_{3}$ [29]

Despite obvious difference in the origin of freevolume defects in crystalline and amorphous materials [3], this direct $\tau_{2}-R(V)$ correlation Eq. (4) is undoubtedly the most correct way to calculate the realistic positrontrapping free volumes in ChVS. It is worth mentioning that defect-related positron lifetimes estimated in respect to Eq. (4) are well correlated with these values in other types of chalcogenides, as it follows from simple comparison with the known data for II-VI crystals gathered by R. Krause-Rehberg and H.S. Leipner (see monograph [1] and literature therein).

So, it seems as fully misleading an attempt to reject this approach in [23] by applying a concept of relaxed geometry for generated free-volume defects in ChVS. This concept (realized also within the DFT approach) occurs to be quite successful for large vacancy clusters in high-symmetric crystalline environment. In this case, the electron density is more affected by localized positrons and resulting transition to relaxed geometry of defects becomes necessary. Thus, non-self-consistent electronic calculations for multi-vacancies in Si crystals performed under LMTO-ASA (linear-muffin-tin-orbital atomic-sphere-approximation) Green's-function method lead to systematic reduction in positron lifetimes for tetra- and larger vacancies as compared with the unrelaxed case [36]. In other words, the realistic free volumes $V$ responsible for positron trapping with defect lifetime $\tau_{2}$ becomes underestimated (e.g. reduced, but not enhanced as it argued in $[22,23]$ ) in some crystalline lattices. But, this effect can apparently be neglected in isotropic glass and low-symmetric crystalline matrices, where multi-atomic high-symmetric defect configurations are rather impossible.

\section{Modified master equation for $\tau_{2}-R$ correlation in As-S/Se glasses}

It seems important to note that nobody before has proved an identity of positron and o-Ps trapping in ChVS, since typical content of the latter does not exceed the experimentally detectable limit of $\sim 2 \%$ [3]. Nevertheless, we can further use the generalized description through Eq. (1) to find possible correlations between defect-related positron lifetimes $\tau_{2}$ and responsible void radii $R$ (or volumes $V$ in a spherical approximation), employing a wide range of known experimental data for different ChVS systems (such as those tabulated in the monograph [3]). The DFTcalculations of Jensen's et al. [29] for As-Se chalcogenides can serve as a justification for these correlations.

So let's assume that compositional variations in defect-related positron annihilation lifetime $\tau_{2}$ are the same as those in Ps lifetime $\tau_{3}$, so we can apply generalized Eq. (1) to determine the corresponding free volumes in ChVS. Of course, such consideration is valid only for isostructural ChVS possessing similar type of glass-forming structural units. So, positrons become indeed trapped similarly to Ps atoms (which is a 
necessary condition to validate the sequent $\tau_{3}-\tau_{2}-R$ estimation procedure [16]) only in essentially narrow range of ChVS compositions of the same structural bonding. In case of $\mathrm{As}-\mathrm{S}(\mathrm{Se})$ glasses built of $\mathrm{AsS} / \mathrm{Se}_{3}$ pyramids interlinked with Se-chains of different lengths $[3,11]$, this is valid near stoichiometric $\mathrm{g}-\mathrm{As}_{2} \mathrm{~S}(\mathrm{Se})_{3}$ stretched mainly in S/Se-rich side [7,8]. Fortunately, to parameterize Eq. (1) under these conditions, we can conveniently use $\mathrm{g}-\mathrm{As}_{2} \mathrm{~S}(\mathrm{Se})_{3}$ as high-reliable testing probes of layered ChVS [4] with experimental positron trapping modes given in Table 1 . The corresponding free volumes of trapping voids for these specimens can be estimated on the basis of previous DFT calculations of K.O. Jensen et al. [29]. With above Eq. (4) applied to defect-related lifetimes $\tau_{2}$, the corresponding radii $R$ of free-volume trapping voids occur to be 2.92 and $2.82 \AA$ for $\mathrm{g}-\mathrm{As}_{2} \mathrm{Se}_{3}$ and $\mathrm{g}-\mathrm{As}_{2} \mathrm{~S}_{3}$, respectively (see Table 1).

By using the further correlation equation between the lifetime $\tau_{2}$ and free-volume radius $R$ in its generalized form (1) with characteristic values of $C$ equal to the intrinsic bulk positron lifetime $\tau_{b}$ as given in Table 1, we can easily estimate the electron layer thickness $\Delta R$ for positron penetration wall region of the voids. This $C$ parameter (included also in Table 1) occurs to be 5.15 and $5.73 \AA$ for $g-\mathrm{As}_{2} \mathrm{Se}_{3}$ and g-As $\mathrm{S}_{3}$, respectively. These estimations seem to be quite realistic, if we accept that in case of $\mathrm{ChG}$ the most effective positron traps represent themselves as geometrically arrested free volumes surrounded by BFSA emerging from neighboring chalcogen atoms at the bottom of $\mathrm{AsS} / \mathrm{Se}_{3}$ pyramids [3, 4, 8]. Being involved into heteropolar covalent bonding between chalcogen ( $\mathrm{S}, \mathrm{Se})$ and pnictogen (As) atoms possessing different electronegativities [3, 28], these BFSA carry an effective negative charge forming relatively thick walls of free-volume voids.

Schematic illustration for the voids in the layeredtype structure of $c-\mathrm{As}_{2} \mathrm{~S}_{3}$ showing arrested geometrical free volumes (yellow colored) created by neighboring interlinked negatively-charged BFSA between two layers of chained $\mathrm{AsS}_{3 / 2}$ pyramids is given in Fig. 1. The semi-spheres of high-electron densities around As atoms $\left(\mathrm{AsS}_{3 / 2}\right.$ pyramids) are dark-grey colored, while the BFSA of low-electron density cones around S atoms are shown by light-grey color. Because of such topology of network-forming structural units, the central part of interlayer space occurs to be free of atoms forming a geometrical free volume (which is accessible for other atoms introduced in a glassy network) surrounded by free volumes of neighboring BFSA. Thus, the positron trapping centers in ChVS possess a specific structure being composed of geometrical free volumes surrounded by free-volume shell of BFSA, the input of latter being decisive. Since chemical environment of these voids is almost the same for chalcogen-enriched $\mathrm{g}-\mathrm{As}_{\mathrm{x}} \mathrm{S}(\mathrm{Se})_{100-\mathrm{x}}$ from stoichiometric $(x=40)$ to approximately $x=20$ compositions, where the BFSA based on one- and two-atom chalcogen chains
$(=\mathrm{As}-\mathrm{S}-\mathrm{As}=$ and $=\mathrm{As}-\mathrm{S}-\mathrm{S}-\mathrm{As}=$ bridges $)$ dominate in outer wall of the voids $[3,8,37]$, this fully explains the observed compositional variation in defect-related positron lifetimes $\tau_{2}$ [7]. Effect of Ps quenching or/and inhibition due to interaction with chemical environment is expected to be maximal in this compositional domain. In terms of positron annihilation [1,2], it means that surface trapping states prefer over volume ones just in this range of ChVS compositions.

This circumstance is a decisive in our consideration, justifying the constancy of $\Delta R$ value as determined for $\mathrm{g}-\mathrm{As}_{2} \mathrm{~S}(\mathrm{Se})_{3}$ (Table 1) to calibrate generalized master Eq. (1) for similar ChVS characterized by the same type of positron traps. We performed this calibration procedure on the basis of known experimental positron trapping modes $[3,4,7,8$, $34,35]$ and void radii calculated in respect to Eq. (4) for 13 chalcogen-enriched compositions of $\mathrm{g}-\mathrm{As}_{\mathrm{x}} \mathrm{S}(\mathrm{Se})_{100-\mathrm{x}}$ (see Table 2).

The resulting full set of experimental $\tau_{2}-R$ data for $\mathrm{g}-\mathrm{As}_{\mathrm{x}} \mathrm{S}(\mathrm{Se})_{100-\mathrm{x}}$ is plotted in Fig. 2. It is clearly seen these results are well grouped around monotonically growing steep-like function as in the case of molecular polymers [16-21]. With a satisfactory goodness (determined by averaged value of mean root-square deviations of experimental points from the fitting curve) near 0.90 , these data can be effectively fitted by modified Eq. (1) with parameters $C=0.285 \mathrm{~ns}$ and $\Delta R=$ $5.50 \AA$ :

$$
\tau_{2}=0.285 \cdot\left[1-\frac{R}{R+5.50}+\frac{1}{2 \pi} \cdot \sin \left(\frac{2 \pi R}{R+5.50}\right)\right]^{-1}
$$

or simplified linear approximation in the form of

$\tau_{2}=0.212 \cdot(1+0.244 \cdot R)$

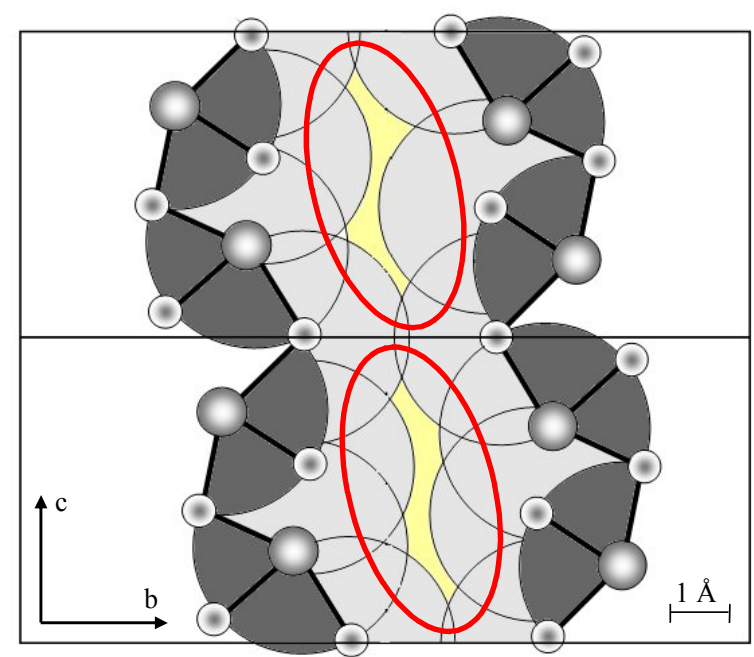

Fig. 1. Schematic illustration of free-volume positron trapping voids (red-distinguished ellipses) in the layered structure ( $b c$ plane) of crystalline c- $\mathrm{As}_{2} \mathrm{~S}_{3}$ (see the text for details). 
Table 2. Experimental positron-trapping modes of $\mathrm{g}-\mathrm{As}_{2} \mathrm{Se}_{3}[4]$ and $\mathrm{g}-\mathrm{As}_{2} \mathrm{~S}_{3}[7,22]$ and corresponding void parameters calculated using Eq. (4) within K.O. Jensen's et al. approach [29].

\begin{tabular}{|c|c|c|c|c|c|c|c|c|c|}
\hline \multirow[t]{2}{*}{ Sample } & \multicolumn{6}{|c|}{ Positron-trapping parameters } & \multicolumn{2}{|c|}{$\begin{array}{l}\text { Void parameters in } \\
\text { Eq. (4) }\end{array}$} & \multirow{2}{*}{$\begin{array}{c}\Delta R \text { in } \\
\text { Eq.(1), } \\
\AA\end{array}$} \\
\hline & $\tau_{1}, \mathrm{~ns}$ & $\tau_{2}, \mathrm{~ns}$ & $I_{2}$, a.u. & $\tau_{a v}, \mathrm{~ns}$ & $\tau_{b}, \mathrm{~ns}$ & $\kappa_{d}, \mathrm{~ns}^{-1}$ & $R, \AA$ & $V, \AA^{3}$ & \\
\hline $\mathrm{g}-\mathrm{As}_{2} \mathrm{Se}_{3}$ & 0.20 & 0.37 & 0.60 & 0.30 & 0.28 & 1.31 & 2.92 & 100 & 5.15 \\
\hline $\mathrm{g}-\mathrm{As}_{2} \mathrm{~S}_{3}$ & 0.19 & 0.36 & 0.75 & 0.32 & 0.29 & 2.01 & 2.82 & 90 & 5.73 \\
\hline
\end{tabular}

Table 3. Compositional changes in positron modes determined within two-state trapping model for g-As $\mathrm{S}_{100-x}[3,8,22]$ and ${\mathrm{g}-A s_{x}} \mathrm{Se}_{100-x}[3,4,8,34,35]$.

\begin{tabular}{|c|c|c|c|c|c|c|}
\hline Glass composition & $\tau_{1}, \mathrm{~ns}$ & $\tau_{2}, \mathrm{~ns}$ & $I_{2}$, a.u. & $\tau_{a v}, \mathrm{~ns}$ & $\tau_{b}, \mathrm{~ns}$ & $R, \AA$ \\
\hline $\mathrm{As}_{18} \mathrm{~S}_{82}$ & 0.192 & 0.342 & 0.75 & 0.304 & 0.286 & 2.59 \\
\hline $\mathrm{As}_{22} \mathrm{~S}_{78}$ & 0.191 & 0.345 & 0.74 & 0.304 & 0.285 & 2.68 \\
\hline $\mathrm{As}_{25} \mathrm{~S}_{75}$ & 0.196 & 0.345 & 0.74 & 0.306 & 0.287 & 2.63 \\
\hline $\mathrm{As}_{28} \mathrm{~S}_{72}$ & 0.195 & 0.346 & 0.75 & 0.308 & 0.290 & 2.58 \\
\hline $\mathrm{As}_{33} \mathrm{~S}_{67}$ & 0.205 & 0.362 & 0.65 & 0.308 & 0.287 & 2.97 \\
\hline $\mathrm{As}_{36} \mathrm{~S}_{64}$ & 0.193 & 0.351 & 0.73 & 0.309 & 0.289 & 2.71 \\
\hline $\mathrm{As}_{40} \mathrm{~S}_{60}$ & 0.193 & 0.361 & 0.71 & 0.313 & 0.289 & 2.90 \\
\hline $\mathrm{As}_{28} \mathrm{Se}_{72}$ & 0.200 & 0.331 & 0.70 & 0.291 & 0.276 & 2.35 \\
\hline $\mathrm{As}_{30} \mathrm{Se}_{70}$ & 0.209 & 0.336 & 0.66 & 0.293 & 0.278 & 2.40 \\
\hline $\mathrm{As}_{35} \mathrm{Se}_{65}$ & 0.200 & 0.339 & 0.69 & 0.296 & 0.279 & 2.43 \\
\hline $\mathrm{As}_{36} \mathrm{Se}_{64}$ & 0.188 & 0.343 & 0.72 & 0.300 & 0.279 & 2.51 \\
\hline $\mathrm{As}_{38} \mathrm{Se}_{62}$ & 0.190 & 0.342 & 0.73 & 0.301 & 0.281 & 2.45 \\
\hline $\mathrm{As}_{40} \mathrm{Se}_{60}$ & 0.200 & 0.370 & 0.60 & 0.300 & 0.280 & 2.92 \\
\hline
\end{tabular}

The above master Eqs. (5) and (6) give with a reasonable precision the correlation relationships for void radii $R$ in As chalcogenides within 2.3-3.0 $\AA$ range. At present, it is difficult to extend them beyond these boundaries as well as for other ChVS (such as Ge chalcogenides) because of lack of available experimental data. Difference between Eq. (2) for $\tau_{2}-R$ correlation in typical polymers as calibrated in [16] (shown by bluecolored dashed line in Fig. 2) and Eq. (5) corresponding to the same correlation in As- $\mathrm{S} / \mathrm{Se}$ glasses is obvious. Thus, the positron-trapping void volume $V$ proper to $\tau_{2}=$ $0.36 \mathrm{~ns}$ in $\mathrm{g}-\mathrm{As}_{2} \mathrm{~S}_{3}$ was estimated to be $98 \AA^{3}$ (in a spherical approximation for $R=2.86 \AA$ ) that is nearly twice higher than $56.7 \AA^{3}$ in molecular polymers (see Table 1), as it follows from Eq. (2).

So, in case of $\mathrm{g}-\mathrm{As}_{\mathrm{x}} \mathrm{S}(\mathrm{Se})_{100-\mathrm{x}}$ examined through modified master Eqs. (5) and (6), we deal with the systematically enhanced void radii $R$ in respect to molecular polymers [16-21]. The corresponding weakened interaction between positrons and void environment is thought to be due to partial transition from volume to surface trapping states, the process which is characteristic for large vacancy clusters [38] like those outlined in Fig. 1.

With further decrease in the void radius $R$ in $\mathrm{g}$ $\mathrm{As}_{\mathrm{x}} \mathrm{S}(\mathrm{Se})_{100-\mathrm{x}}$ at $x<20$, the type of preferential positron traps significantly changes. Free volumes emerging from overlapping BFSA of homopolar chalcogen-chalcogen bonds having preferentially neutral environment become dominant in positron trapping. These voids do not participate in Ps quenching or inhibition like those having more negatively-charged environment in near- stoichiometric ChVS. This leads to steep decrease in the values of defect-related positron lifetimes $\tau_{2}$. As a result, the left part of $\tau_{2}-R$ curve (not shown in Fig. 2) demonstrates a characteristic trend towards lower lifetimes $\tau_{2}$, the similar behavior being observed also in polymers [16-21], vacancy clusters in metals [38-42] and semiconductors [43-45]. This good coincidence between experimental and theoretically estimated positron trapping modes for g$\mathrm{As}_{x} \mathrm{~S}(\mathrm{Se})_{100-x}$ should not be taken too seriously, since the $\tau_{2}-R$ correlation Eq. (1) serves only as an estimation one to keep realistic tendencies in free-volume evolution, a full similarity in the behavior of $\tau_{3}$ and $\tau_{2}$ lifetimes being decisive condition dependent on material [16]. Whichever the case, one should be careful by approaching Eqs. (2) and (3) for other types of materials possessing principally different type of void environment as compared to molecular substrates, such as polymers.

Fundamental importance of this comparison can be well illustrated graphically by diagram in Fig. 3, which reproduces known generalization for great amount of chemically-different PAL-tested materials [1-3]. Typical positron lifetimes are strongly ranged from metals and metallic alloys to high-porous substances such as mezoporous materials (zeolites) and special glasses prepared by mass destruction (creation of developed inner pore structure due to etching of some separated phases). ChVS are entirely fit in this scheme with semiconductors group, being just after metals but beyond typical polymers (with really very slight overlapping region). It means that any analytical correlation validated for polymers and/or zeolites will be with a necessity underestimating as applying for ChVS. 


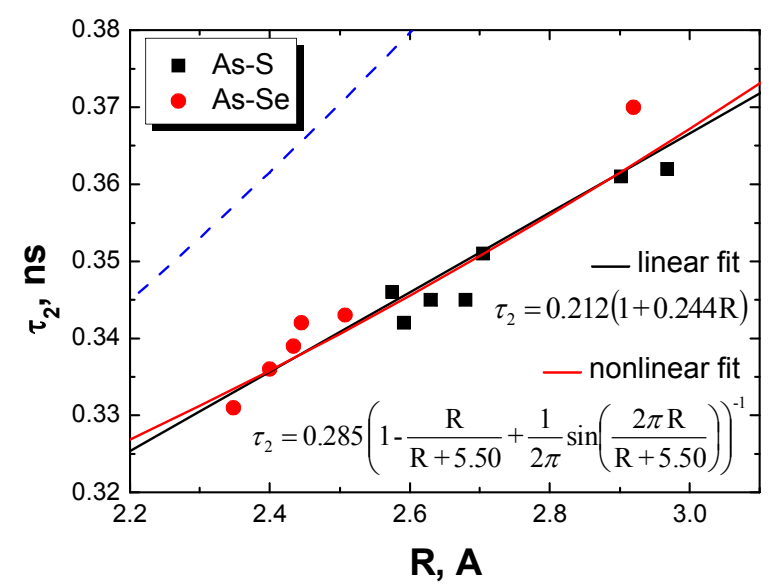

Fig. 2. Experimental $\tau_{2}-R$ plot for $g-\mathrm{As}_{x} \mathrm{~S}_{100-x}$ (black squares, $18 \leq x \leq 40$ ) and $g-\mathrm{As}_{x} \mathrm{Se}_{100-x}$ (red circles, $28 \leq x \leq 40$ ) fitted with linear Eq. (6) and nonlinear Eq. (5). The blue-colored dashed line corresponds to Eq. (2) calibrated for typical polymers in [16].

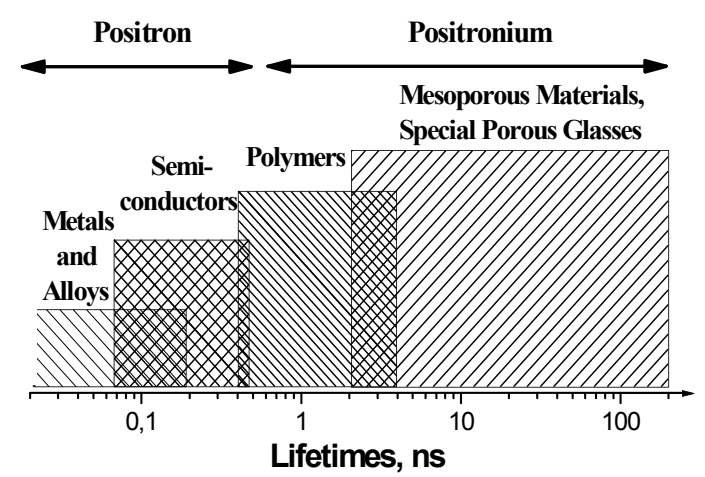

Fig. 3. Scale of typical positron and Ps lifetimes in different types of chemically-specific materials.

By finishing, it seems noteworthy to underline that all above procedures transforming distribution of positron lifetimes into distribution of void radii are derived within a simple spherical approximation, an approach that neglects any non-spherical deviation in the void shape. Since surface of free volume is minimal for a sphere, the corresponding defect-related positron lifetimes $\tau_{2}$ are maximal [46]. It means that void volumes determined within any of estimation procedures are rather underestimated as compared with realistic volumes responsible for positron and Ps trapping.

\section{Conclusions}

The modified master equations in the form of sequent $\tau_{3^{-}}$ $\tau_{2}-R$ correlation

$\tau_{2}=0.285 \cdot\left[1-\frac{R}{R+5.50}+\frac{1}{2 \pi} \cdot \sin \left(\frac{2 \pi R}{R+5.50}\right)\right]^{-1}$

or in a simplified linear approximation form $\tau_{2}=0.212 \cdot(1+0.244 \cdot R)$

allow unambiguous evaluation of the void radii $R$ (or free volumes $V$ in a spherical approximation) in ChVS of As-S(Se) type on the basis known defect-related positron annihilation lifetimes $\tau_{2}$. This approach is consistent with predictions of the DFT calculation procedure of direct solving the Schrödinger equation for adopted electron-positron interaction model justified for orthorhombic $\mathrm{As}_{2} \mathrm{Se}_{3}$. The specific chemical environment of free-volume voids possessing an effective negative charge owing to difference in the electronegativities of covalent chemical bonds belonging to neighbouring network-forming polyhedrons is shown to play decisive role in the governed PAL-sensitive structure-properties correlations in different ChVS systems. Speculative attempt to treat positron trapping modes in ChVS like as in typical molecular substrates such as polymers leads actually only to misleading conclusions, in obvious contrast to experimentally credible model of positron and Ps trapping developed on the basis of proved PAL data.

\section{References}

1. R. Krause-Rehberg, H.S. Leipner, Positron Annihilation in Semiconductors. Defect Studies. Springer-Verlag, Berlin-Heidelberg-New York, 1999.

2. Y.C. Jean, P.E. Mallon, D.M. Schrader, Principles and Application of Positron and Positronium Chemistry. World Sci. Publ. Co. Pte. Ltd., New Jersy-London-Singapore-Hong Kong, 2003.

3. O. Shpotyuk, J. Filipecki, Free Volume in Vitreous Chalcogenide Semiconductors: Possibilities of Positron Annihilation Lifetime Study. Ed. WSP, Czestochowa, 2003.

4. M. Hyla, J. Filipecki, O. Shpotyuk, M. Popescu, V. Balitska, Stoichiometric arsenic sulphoselenides as testing probes for positron trapping in chalcogenide glasses // J. Optoelectron. Adv. Mat. 9, p. 31773181 (2007).

5. O.I. Shpotyuk, J. Filipecki, V.O. Balitska, Radiation-induced extended free-volume defects in mixed ternary Ge-As/Sb-S glasses studied with PALS technique // J. Optoelectron. Adv. Mat. 10, p. 3193-3197 (2008).

6. O. Shpotyuk, J. Filipecki, M. Hyla, A. Ingram, Critical comments on speculations with open and closed "free volume defects ... in ion-conducting $\mathrm{Ag} / \mathrm{AgI}-\mathrm{As}_{2} \mathrm{~S}_{3}$ glasses..." // Solid State Ionics, 208, p. 1-3 (2012).

7. V. Balitska, Ya. Shpotyuk, J. Filipecki, O. Shpotyuk, M. Iovu, Post-irradiation relaxation in vitreous arsenic/antimony trisulphides // J. NonCryst. Sol. 357, p. 487-489 (2011).

8. A. Ingram, R. Golovchak, M. Kostrzewa, S. Wacke, M. Shpotyuk, O. Shpotyuk, Compositional depen- 
dences of average positron lifetime in binary AsS/Se glasses // Phys. B, 407, p. 652-655 (2012).

9. O. Shpotyuk, R. Golovchak, A. Ingram, V. Boyko, L. Shpotyuk Comparative study of extended freevolume defects in As- and Ge-based glassy semiconductors: theoretical prediction and experimental probing with PAL technique // Phys. Status Solidi (C), 10, p. 117-120 (2013).

10. R. Golovchak, A. Ingram, S. Kozyukhin, O. Shpotyuk, Free volume fragmentation in glassy chalcogenides during natural physical ageing as probed by PAL spectroscopy // J. Non-Cryst. Solids, 377, p. 49-53 (2013).

11. A. Feltz, Amorphous and Vitreous Inorganic Solids. Mir, Moscow, 1986.

12. X.H. Zhang, B. Bureau, P. Lucas, C. BoussardPledel, J. Lucas, Glasses for seeing beyond visible // Chem. Eur. J. 14, p. $432-442$ (2008).

13. B.J. Eggleton, Chalcogenide photonics: fabrication, devices, and applications // Opt. Express, 18, p. 26632-26634 (2010).

14. A. Zakery, S.R. Elliott, Optical Non-linearities in Chalcogenide Glasses and Their Applications. Springer-Verlag, Berlin-Heidelberg, 2007.

15. A.B. Seddon, Z. Tabg, D. Furniss, S. Sujecki, T.M. Benson, Progress in rare-doped mid-infrared fiber lasers // Opt. Express, 18, p. 26704-26719 (2010).

16. K.S. Liao, H. Chen, S. Awad, J.P. Yuan, W.S. Hung, K.R. Lee, J.Y. Lai, C.C. Hu, Y.C. Jean, Determination of free-volume properties in polymer without orthopositronium components in positron annihilation lifetime spectroscopy // Macromolecules, 44, p. 6818-6826 (2011).

17. Q. Deng, C.S. Sundar, Y.C. Jean, Pressure dependence of free-volume hole properties in an epoxy polymer // J. Phys. Chem. 96, p. $492-495$ (1992).

18. Q. Deng, Y.C. Jean, Free-volume distributions of an epoxy polymer probed by positron annihilation: pressure dependence // Macromolecules, 26, p. 3034 (1993).

19. Q. Deng, F. Zandiehnadem, Y.C. Jean, Freevolume distributions of an epoxy polymer probed by positron annihilation: temperature dependence // Macromolecules, 25, p. 1090-1095 (1992).

20. Y.C. Jean, Positron annihilation in polymers // Mat. Sci. Forum, 175-178, p. 59-70 (1995).

21. R. Zhang, Y.C. Wu, H. Chen, J. Zhang, Y. Li, T.C. Sandreczki, Y.C. Jean, Chemical environment in halogenated styrene polymers studied by using positron annihilation lifetime spectroscopy // Rad. Phys. Chem. 68, p. 481-484 (2003).

22. T. Kavetskyy, J. Borc, P. Petkov, K. Kolev, T. Petkova, V. Tsmots, Reply on the "critical comments on speculations with ... free-volume defects $\ldots$ in ion-conducting $\mathrm{Ag} / \mathrm{AgI}-\mathrm{As}_{2} \mathrm{~S}_{3}$ glasses..." // Solid State Ionics, 233, p. 107-109 (2013).

23. T. Kavetskyy, Modified correlation equation in the FSDP-related void-based model for $\mathrm{As}_{2} \mathrm{~S}(\mathrm{Se})_{3}$ chalcogenide glass // Semiconductor Physics,
Quantum Electronics \& Optoelectronics, 16, p. 136-139 (2013).

24. S.J. Tao, Positronium annihilation in molecular substances // J. Chem. Phys. 56, p. 5499-5510 (1972).

25. M. Eldrup, D. Lightbody, J.N. Sherwood, The temperature dependence of positron lifetimes in solid pivalic acid // Chem. Phys. 63, p.51-58 (1981).

26. V.I. Grafutin, I.N. Meshkov, E.P. Prokop'ev, N.O. Khmelevskii, S.L. Yakovenko, Determination of the size of vacancy-type defects in angstrom ranges by positron annihilation spectroscopy // Rus. Microelectronics, 40, p. 428-435 (2011).

27. M. Kastner, Compositional trends in the optical properties of amorphous lone-pair semiconductors // Phys. Rev. B, 7, p. 5237-5252 (1973).

28. L. Pauling, The Nature of the Chemical Bond. Cornell Univ. Press, Ithaca, 1960.

29. K.O. Jensen, P.S. Salmon, I.T. Penfold, P.G. Coleman, Microvoids in chalcogenide glasses studied by positron annihilation // J. Non-Cryst. Solids, 170, p. 57-64 (1994).

30. M. Sob, H. Sormann, J. Kuriplach, Theoretical calculations of positron annihilation characteristics in inorganic solids - recent advantages and problems // Adv. Quant. Chem. 42, p. 77-108 (2003).

31. M.J. Puska, Theoretical aspects of positrons in imperfect solids // Phys. Status Solidi (A), 102, p. 11-29 (1987).

32. M.J. Puska, R.M. Nieminen, Theory of positrons in solids and on solid surfaces // Rev. Mod. Phys. 66, p. 841-897 (1994).

33. M.J. Puska, S. Makinen, M. Manninen, R.M. Nieminen, Screening of positrons in semiconductors and insulators // Phys. Rev. B, 39, p. 7666-7679 (1989).

34. O.K. Alekseeva, V.I. Mihailov, A.P. Chernov, V.P. Shantarovich, Point structural defects studied in chalcogenide semiconductors by positron annihilation // Fizika Tverd. Tela, 19, p. 3452-3454 (1977).

35. O.K. Alekseeva, V.I. Mihajlov, V.P. Shantarovich, Positron annihilation in point defects of the glassy As-Se system // Phys. Status Solidi (A), 48, p. K169-K173 (1978).

36. M. Saito, A. Oshiyama, Lifetimes of positrons trapped at Si vacancies // Phys. Rev. B, 53, p. 78107814 (1996).

37. R. Golovchak, A. Kovalskiy, A.C. Miller, H. Jain, O. Shpotyuk, Structure of Se-rich As-Se glasses by high-resolution X-ray photoelectron spectroscopy // Phys. Rev. B, 76, 125208-1-7 (2007).

38. R.M. Nieminen, J. Laakkonen, Positron trapping rate into vacancy clusters // Appl. Phys. 20, p. 181184 (1979).

39. P. Hautojarvi, J. Heinio, M. Manninen, R. Nieminen, The effect of microvoid size on 
positron annihilation characteristics and residual resistivity in metals // Phil. Mag. 35, p. 973-981 (1977).

40. P. Hautojarvi, Positron annihilation studies of vacancy-type defects // Hyperfine Interactions, 15/16, p. 357-370 (1983).

41. P. Jena, A.K. Gupta, K.S. Singwi, Positron annihilation in small metal voids // Solid State Communs. 21, p. 293-296 (1977).

42. M. Eldrup, B.N. Singh, Studies of defects and defect agglomerates by positron annihilation spectroscopy // J. Nucl. Mat. 251, p. 132-138 (1997).

43. S. Hautakanges, K. Saarinen, L. Liszkay, J.A. Freitas Jr., R.L. Henry, Role of open volume defects in Mg-doped GaN films studied by positron annihilation spectroscopy // Phys. Rev. B, 72, 165303-1-10 (2005).

44. R. Aavikko, K. Saarinen, F. Tuomisto, B. Magnusson, N.T. Son, E. Lanzen, Clustering of vacancy defects in high-purity semi-insulating SiC // Phys. Rev. B, 75, 085208-1-8 (2007).

45. M.J. Puska, C. Corbel, Positron states in Si and GaAs // Phys. Rev. B, 38, p. 9874-9880 (1988).

46. H. Schmitz, F. Muller-Plathe, Calculation of the lifetime of positronium in polymers via molecular dynamics simulation // J. Chem. Phys. 112, p. $1040-1045$ (2000). 